International Journal of Case Reports
(ISSN:2572-8776)

\title{
Multiple septic emboli and myocardial infarction due to vaso- invasive Rhizomucor pusillus infection in a hematologic patient
}

\author{
Pim A. de Ruijter, MD, ${ }^{1}$, Pui-Yuen Lee, MD, ${ }^{2}$,Judith Bonnes, MD, PhD, ${ }^{3}$, Monika G. Looijen- \\ Salamon, MD, ${ }^{2}$, Johannes G. van der Hoeven, MD, PhD, ${ }^{1}$ \\ ${ }^{1}$ Department of Intensive Care, Radboud University Medical Centre Nijmegen, The Netherlands; \\ ${ }^{2}$ Department of Pathology,Radboud University Medical Centre Nijmegen, The Netherlands; \\ ${ }^{3}$ Department of Cardiology, Radboud University Medical Centre Nijmegen, The Netherlands
}

\begin{abstract}
We present a case of a 63-year-old hematologic patient with pulmonary vaso-invasive zygomycosis with Rhizomucor pusillus *Correspondence to Author: after a second stem cell transplantation (SCT) for myelodysPim A. de Ruijter, MD plastic syndrome, complicated by multi organ failure, myocarDepartment of Intensive Care, Raddial ischemia and infarction. Zygomycosis is common in immu- jmegen, The Netherlands nocompromised patients, especially after hematopoietic stem cell transplantation (HSCT). Mucor species have devastating How to cite this article: vaso-invasive properties causing hematogenic dissemination. Pim A. de Ruijter, Pui-Yuen Lee,Antemortem diagnosis may be difficult due to negative cultures. Judith Bonnes, Monika G. LooiDespite adequate treatment outcome tends to be poor. Cardiac jen-Salamon, Johannes G. van zygomycosis is rare. In our patient, the clinical course and imag- der Hoeven. Multiple septic emboli ing results of the myocardial infarction are most consistent with and myocardial infarction due to coronary plaque rupture possibly provoked by severe vaso-invasive pulmonary infection and multi-organ failure.

vaso-invasive Rhizomucor pusillus infection in a hematologic patient. International Journal of Case Reports, 2021 5:227.

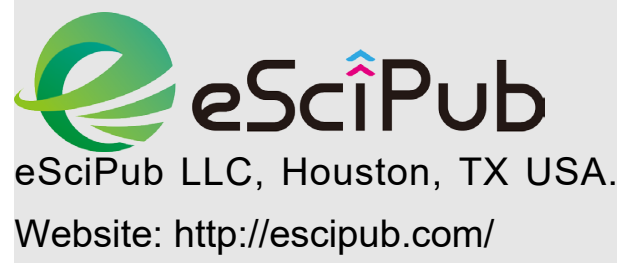




\section{Introduction}

Patients with hematological malignancies are at increased risk for opportunistic infections, especially during treatment with chemotherapy or immunosuppressive therapy. We present a patient with myelodysplastic syndrome (MDS) who developed a pulmonary infection with Rhizomucor pusillus after a second allogenic stem cell transplantation (SCT) with vasoinvasion of the pulmonary vein with multiple septic emboli and myocardial infarction.

\section{Case Report}

63-year-old male presented to our hematology department with a relapse of myelodysplastic syndrome (MDS). Five years earlier he received a successful allogenic stem cell transplantation (SCT) from a HLA-mismatched unrelated donor (MMUD, DQB1 mismatch) for myelodysplastic syndrome (MDS) with rapidly progressive myelofibrosis (JAK-2/ASXL-1 mutation positive) and B-symptoms. He was in molecular remission with JAK2 being negative.

Seven months before presentation, he developed thrombocytopenia. Bone marrow biopsy showed severe aplasia in concordance with hypoplastic MDS. Screening echocardiography showed a normal left ventricular ejection fraction. Pulmonary function tests revealed a mild obstructive lung disease with decreased diffusion capacity.

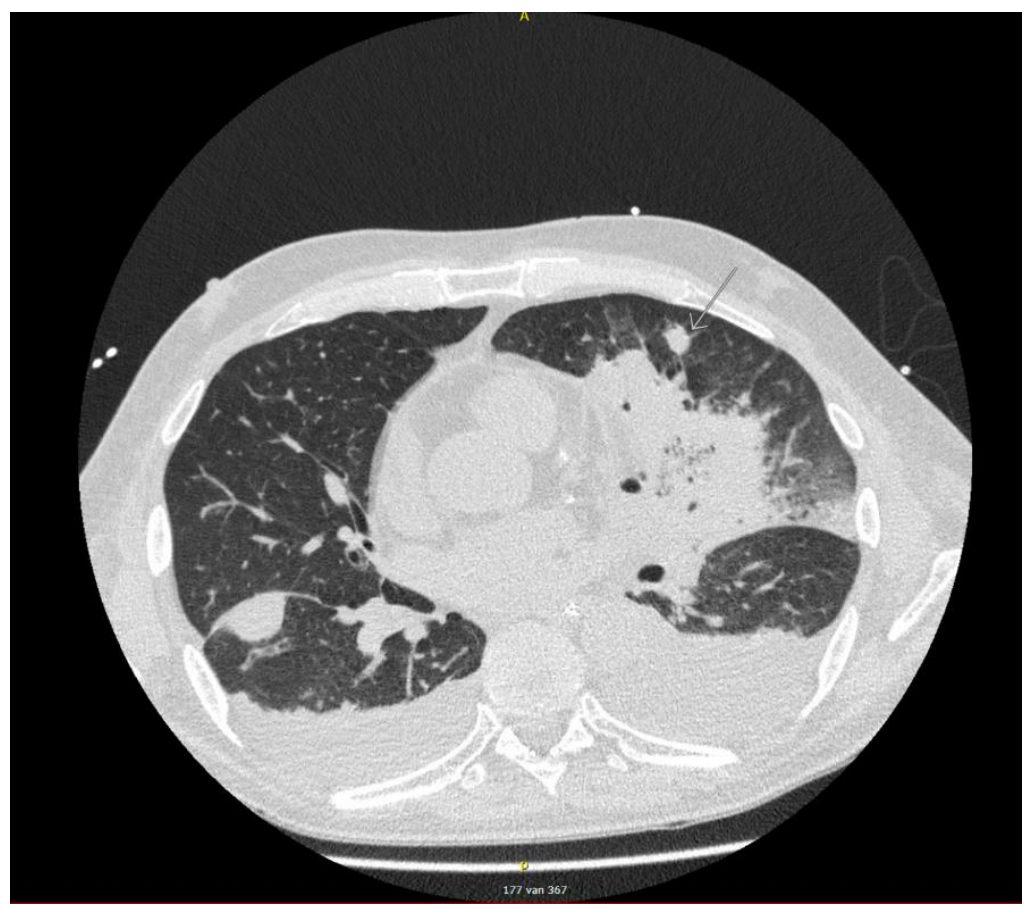

Figure 1: CT-scan of the chest showing bilateral pleural effusions and consolidations in the left lung.

He received an allogenic stem cell retransplantation with Treosulfan-FludarabineATG (Genzyme) preconditioning. On day 1 after the SCT he developed neutropenic fever and ceftazidime was started. A chest CT on day 6 showed perihilar consolidations with postobstruction atelectasis in the left lung with pleural effusion. Multiple focal hypodensities were seen in the spleen, consistent with infarction. A repeat chest CT on day 9 showed progression of consolidations and ground glass pattern and a halo sign in the left upper lobe and right upper and lower lobes (Figure 1). There was minimal pericardial effusion. Amfo B was started at $3 \mathrm{mg} / \mathrm{kg} /$ day because of suspicion of an angio-invasive fungus.

Ten days after the SCT, he was admitted to the ICU because of progressive respiratory distress. He was intubated and a chest X-ray showed bilateral pleural effusions and consolidations in the left lung (Figure 2). A broncho alveolar 
Pim A. de Ruijter et al., IJCR, 2021 5:227

lavage (BAL) was performed. Several petechia PCR for Mucor was positive and cultures were seen in the trachea and lower bronchi. showed rhizomucor pusillus. Respiratory viruses Microscopy showed no hyphae. Aspergillus and TB were all negative. antigen was $<0.1$ and Candida antigen $<5$. A

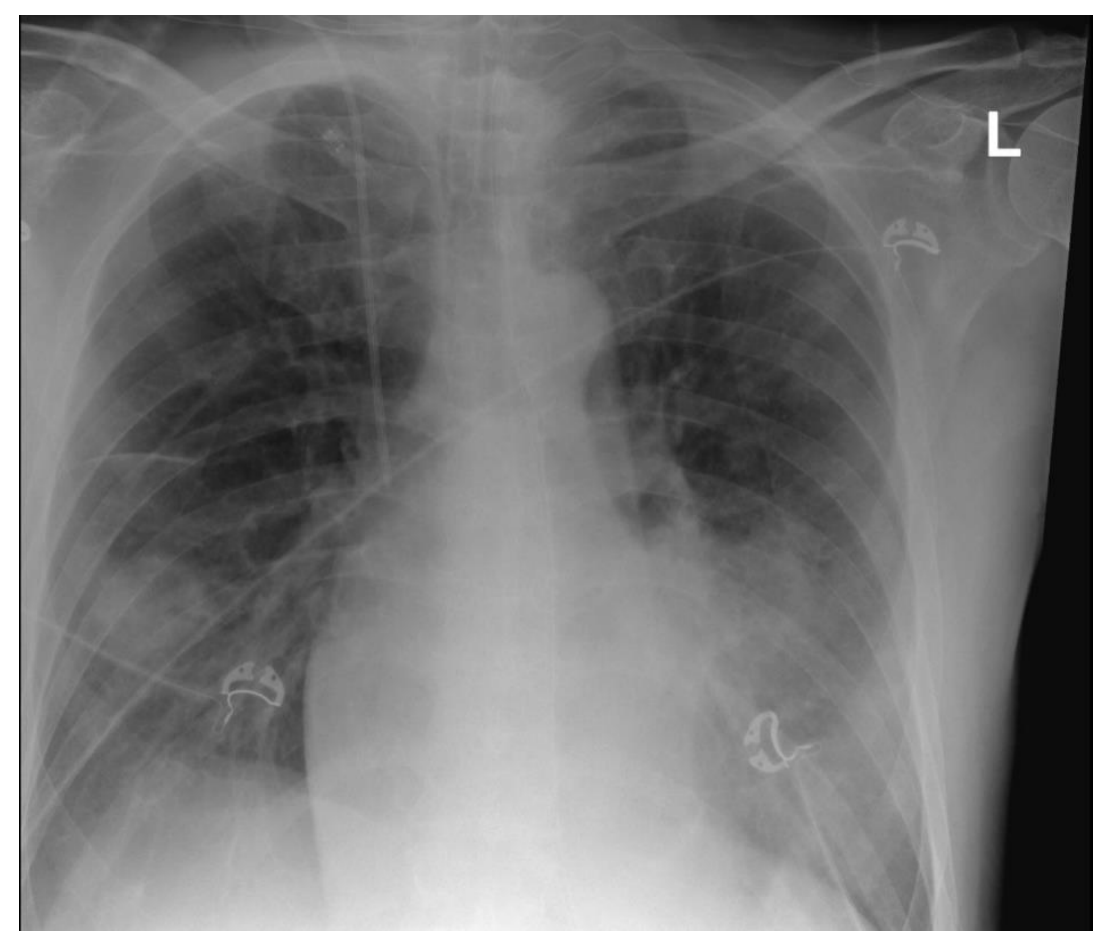

Figure 2: Chest X-ray showing bilateral pleural effusion and consolidation in the left lung.

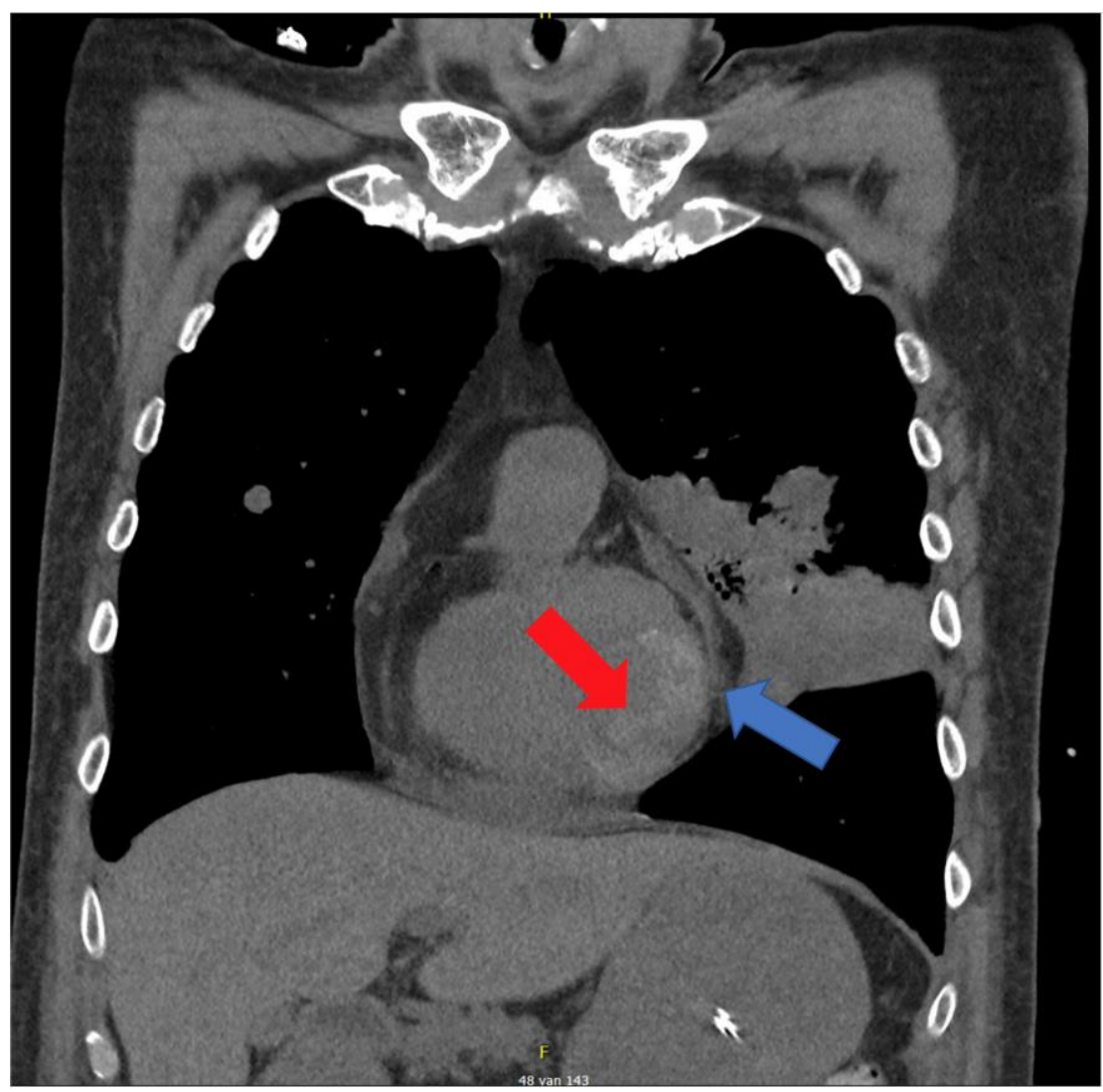

Figure 3A: Coronal view of chest CT showing consolidation in the left lung (blue arrow) in close relation to the pericardium and increased intensity in the myocardium (red arrow). 


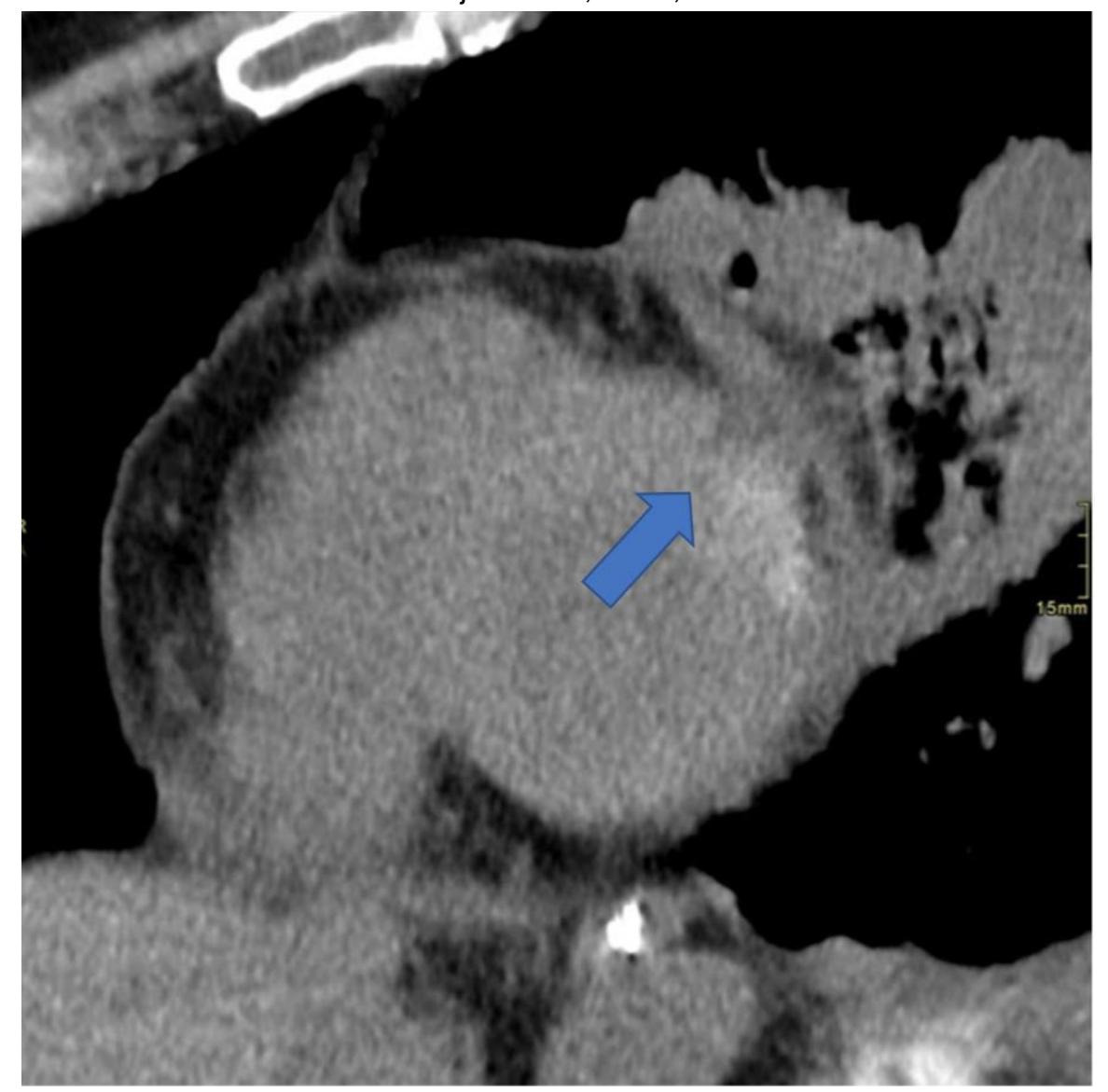

Figure 3B: Sagittal view of Chest CT, detailed view of the heart showing close relation of the pulmonary consolidation with the heart (blue arrow).

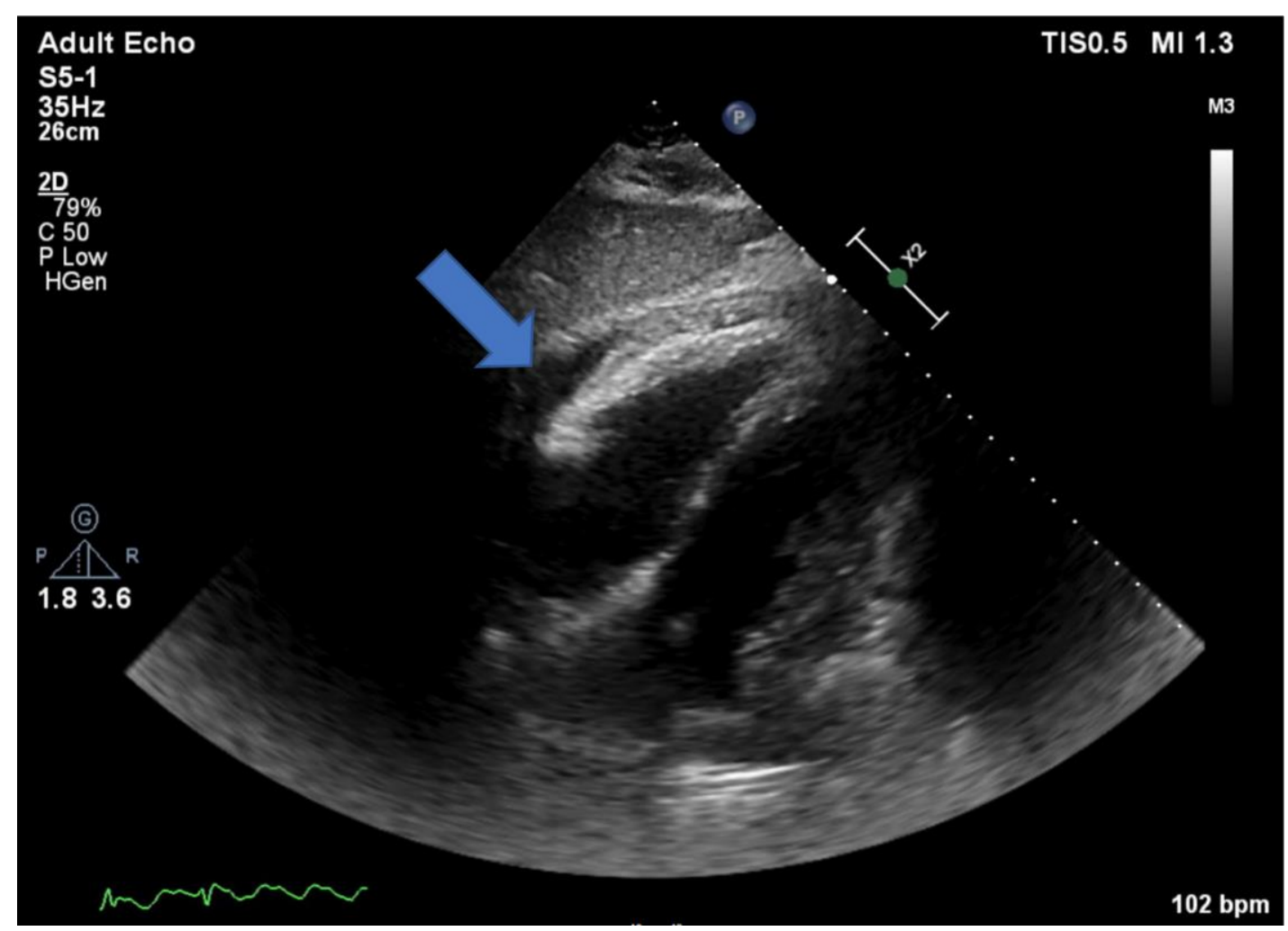

Figure 4: Transthoracic echocardiography, subcostal window, showing prominent epicardial fat and pericardial effusion (blue arrow). 


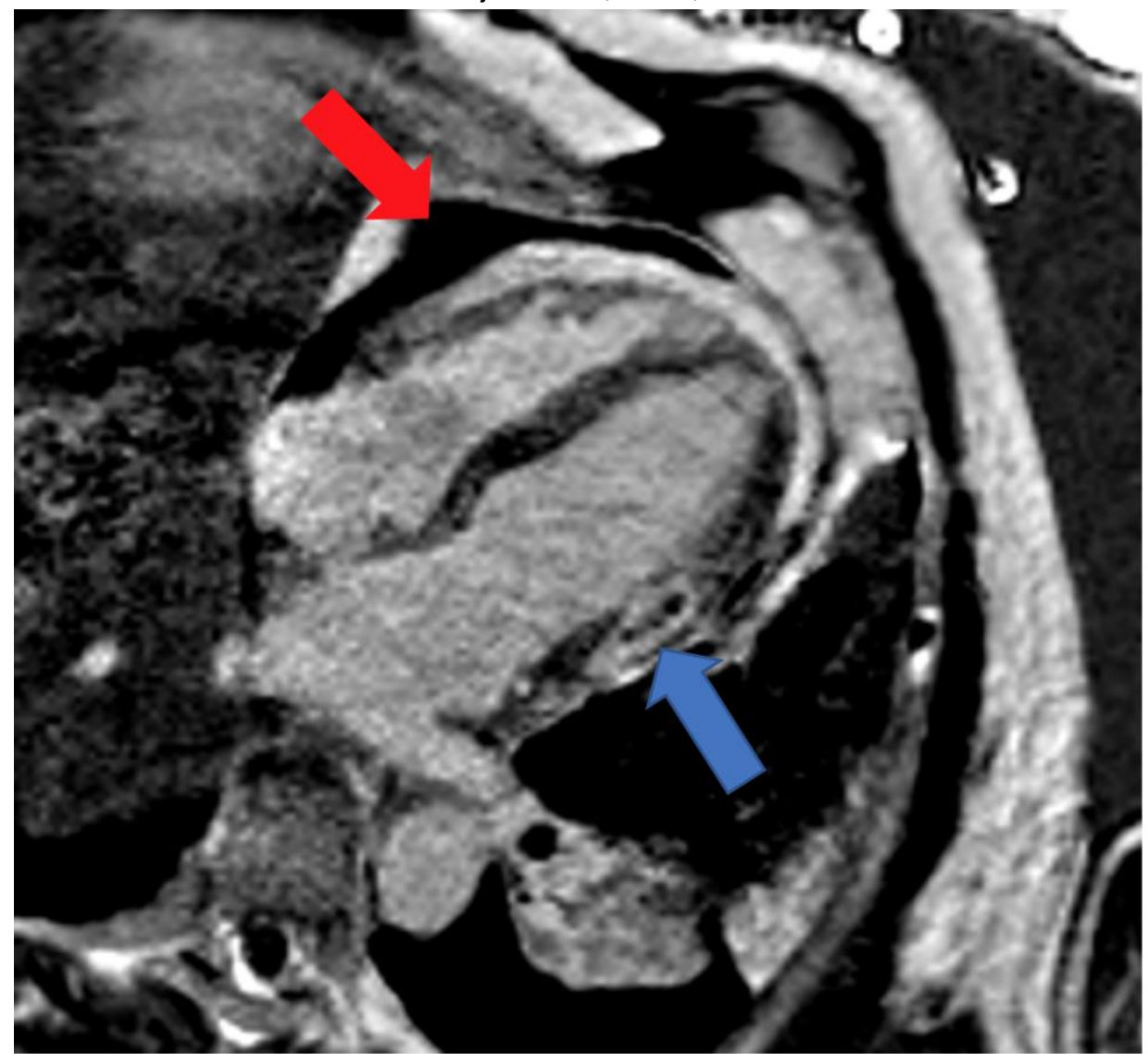

Figure 5: Four chamber view of the heart showing transmural late gadolinium enhancement (bright area - blue arrow) with central microvascular obstruction (dark area - blue arrow) in the bas/mid anterolateral wall compatible with myocardial infarction in the area supplied by the circumflex artery. Prominent epicardial fat (bright - red arrow) and pericardial effusion (black - red arrow) is seen on the right ventricular free wall.
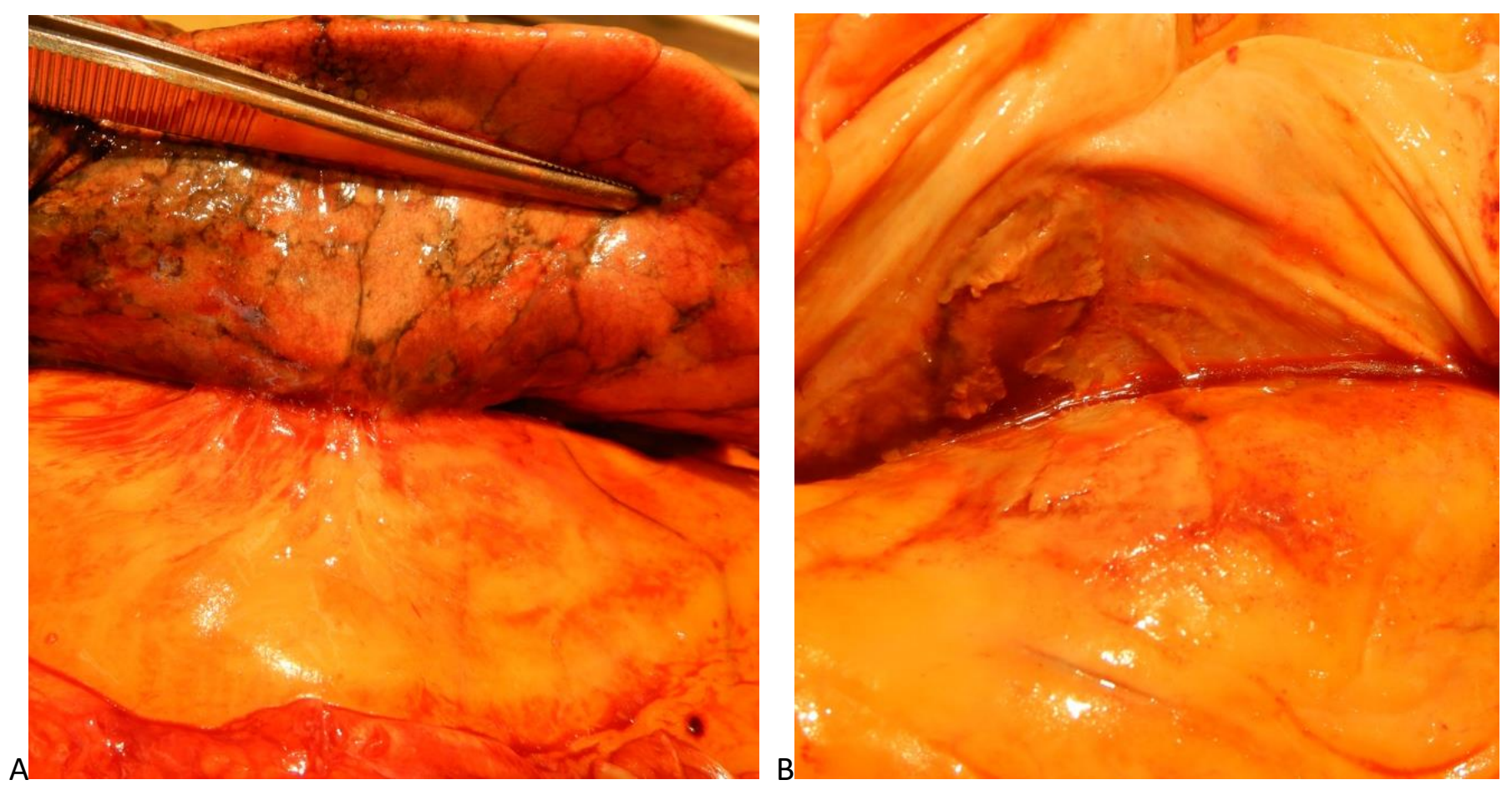

Figure 6: Postmortem examination of the thorax revealed focal adherences of the $(A)$ left lung and pericardium as well of the (B) epi- and pericardium. 

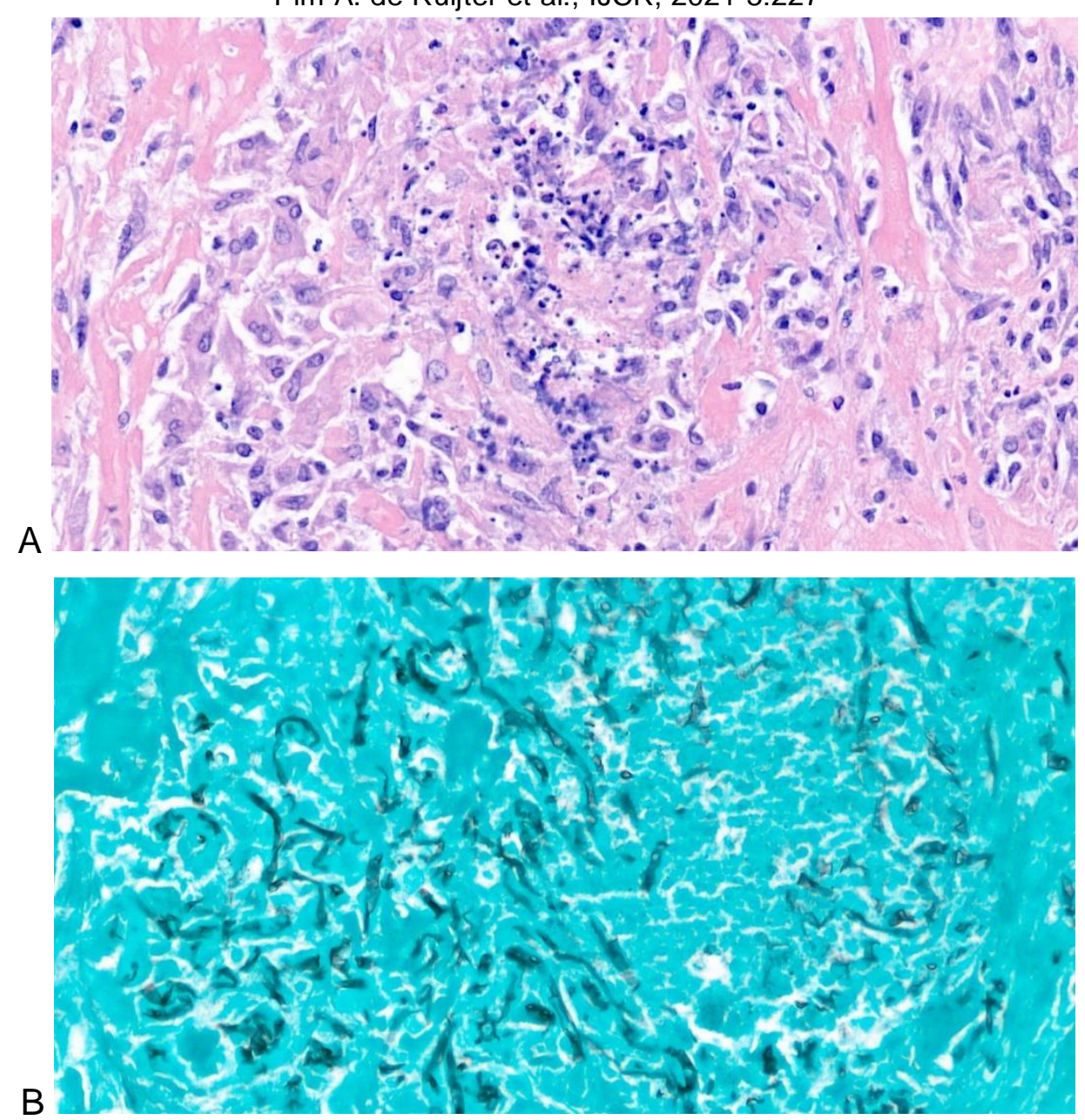

Figure 7: Detail of lung. (A) H\&E-stained section showing acute and chronic inflammation in the lung. (B) Grocott stain of the section demonstrating hyphae and spores.

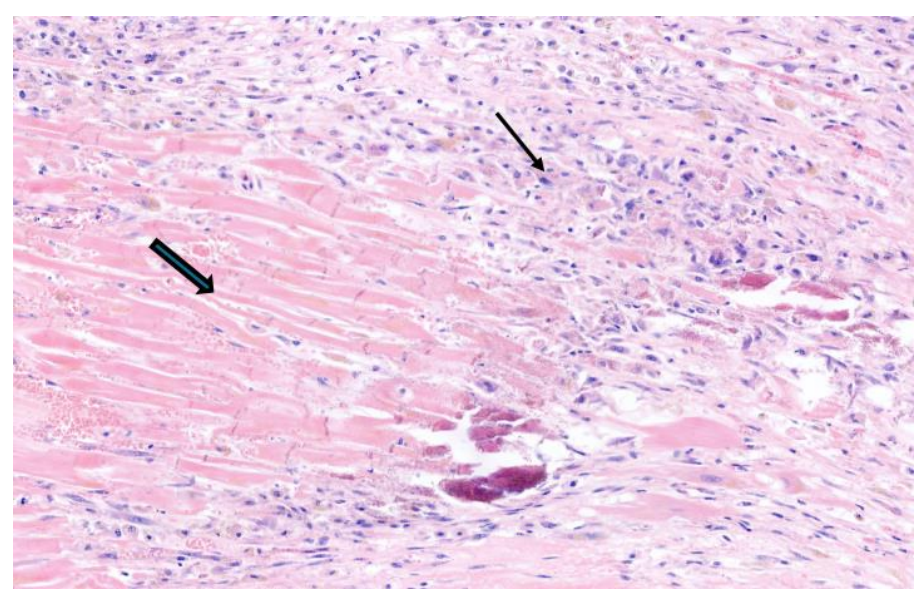

A

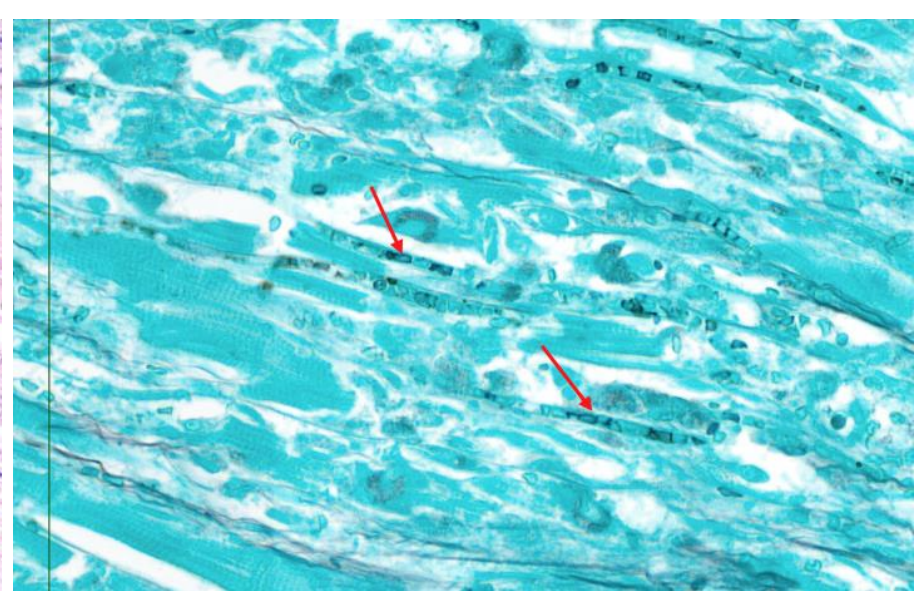

B

Figure 8: Myocardial infarction. (A) H\&E stain (20x magnification) shows recent necrosis with loss of nuclei (thick arrow) surrounded by granulation tissue (thin arrow). (B) Grocott stain (50x) highlights fragments of hyphae.

Isavuconazole was started at $200 \mathrm{mg}$ ttd for 2 elevated liver enzymes. Amfo B was switched to days, followed by $200 \mathrm{mg}$ once daily as micafungin because of acute kidney injury and posaconazole was contraindicated due to increased liver enzymes. 
Prior to ICU admittance (day 7), patient complained of chest pain and the electrocardiogram (ECG) showed new onset atrial fibrillation with rapid ventricular rate for which rate control with digoxin was initiated. Therapeutic anticoagulation was not indicated due to low $\mathrm{CHA}_{2} \mathrm{DS}_{2}$-VASc score and severe thrombocytopenia. A repeated ECG on the next day showed sinus rhythm, but ST segment elevation in the inferolateral ECG leads consistent with transmural myocardial infarction. Invasive coronary angiography was considered but not performed as antithrombotics were regarded contraindicated in the presence of severe thrombocytopenia and increased bleeding tendency. Moreover, cardiac biomarkers were already decreasing. Echocardiography showed a preserved left and right ventricular function without relevant valvular abnormalities.

On day 20 repeated echocardiography was performed and showed a $13 \mathrm{~mm}$ pericardial effusion without clinical or echocardiographic signs of cardiac tamponade and an echogenic structure $(9-10 \mathrm{~mm})$ on the right ventricular free wall (Figure 4).

A chest CT revealed progression of consolidations and post obstruction atelectasis in the lingula (Figure $3 \mathrm{~A}$ and $3 \mathrm{~B}$ ). There was minimal progression of pericardial fluid and a new dense configuration in the left ventricular wall was seen, possibly related to the vasoinvasive Mucor. A subsequent cardiac MRI showed akinesis of the mid antero- and inferolateral wall and apical lateral and inferior wall with ischemic late gadolinium enhancement and central microvascular obstruction consistent with myocardial infarction in the area supplied by the circumflex artery (Figure 5). Invasion of the myocardial wall by adjacent Mucor was not seen. The epicardial density on the right ventricle was due to prominent epicardial fat.

Because of severe vaso-invasive Mucor pneumonia in combination with sepsis and myocardial infarction, treatment was discontinued on day 23 and he died one day later.

Postmortem examination showed vaso-invasive mucor pneumonia. At macroscopic examination, both lungs showed edematous and granular parenchyma consistent with pneumonia and multiple small abscesses. Microscopically, he abscesses consisted of central necrosis containing hyphae and spores, morphologically consistent with mucor spp., surrounded by acute and chronic inflammation (Figure 7). There was obvious angioinvasion: The left upper pulmonary vein showed a thrombus which contained hyphae and partly occluded the lumen.

The pericardial space contained 50cc dark red cloudy fluid. There was fibrinous pericarditis with focal adherences of epi- and pericardium and also focal adherences of the left lung and pericardium (Figure 6 ). The posterior wall of the left ventricle showed a tan-colored area of approximately $5 \times 4 \mathrm{~cm}$, suggesting ischemia. Microscopic investigation confirmed foci of granulation tissue (1-2 weeks of age), in the posterior, lateral and anterior wall of the left ventricle (Figure 8). In addition, the posterior wall and both papillary muscles showed recent necrosis surrounded by very young granulation tissue (less than 1 week of age). In these necrotic areas, again, spores and fragments of hyphae were found. There was no continuous spread of fungi or necrotizing inflammation from the lung into the heart. There was no valvular endocarditis. The coronary arteries showed severe atherosclerosis. Furthermore, recent infarctions were found in the spleen and in the right adrenal glands caused by mycotic emboli.

\section{Discussion}

We present a case of a hematologic patient with pulmonary vaso-invasive zygomycosis with Rhizomucor pusillus after a second SCT for myelodysplastic syndrome, complicated by myocardial ischemia and infarction. We hypothesized that vaso-invasive Mucor may have contributed to the myocardial infarction, by either invasive growth, septic embolies, provoking coronary plaque rupture or type II 
myocardial infarction due to sepsis and multiorgan failure.

Postmortem study did not show continuous inflammation between lungs and pericardium. However, microscopic investigation showed spores and fragments of hyphae in necrotic areas in the heart, spleen and right adrenal gland confirming hematogenic spread causing multi organ failure.

Infections with zygomycetes occur mainly after inhalation of spores through the respiratory tract including the nasal cavities, but also inoculation via disrupted skin in surgical or traumatic wounds occurs. In immunocompetent humans spores are contained and suppressed by macrophages and hypha are killed by neutrophil action. In immunocompromised patients with impaired leukocyte function or neutropenia germination of spores occurs rapidly with development and proliferation of hyphal elements. ${ }^{[1]}$

Common sites for zygomycosis are paranasal sinuses with or without cerebral involvement, pulmonary tract, gastrointestinal tract and skin. In HSCT patients pulmonary localization is the most common site of zygomycosis. ${ }^{[2]}$ Infection with Mucorales is characterized by rapidly progressive severe tissue destruction, necrosis and vaso-invasive growth with dissemination throughout the body and thrombosis. ${ }^{[1,3-5]}$

\section{Cardiac zygomycosis}

Cardiac involvement in zygomycosis has been described in several case reports in immunocompromised patients and post cardiac surgery and is most often part of disseminated zygomycosis. ${ }^{[6-9]}$ Most cases are diagnosed post-mortem, only few case reports of antemortem diagnosis are present in literature. Signs of cardiac involvement include: myocardial infarction, new onset heart failure, endocarditis, valvular insufficiency, pericarditis or rhythm disturbances. ${ }^{[10-13]}$

The formation of thrombi and vaso-invasive properties of zygomycosis are responsible for invasion of endocardium and myocardium.
Acute myocardial infarction has been described due to growth into and obstruction of coronary arteries. Invasion and occlusion of microvessels in the myocardium may lead to infarction. ${ }^{[9,11,13 \text {, }}$ 14] Also after cardiac (transplant) surgery zygomycosis has been described. ${ }^{[6,7,15]}$ Native valve zygomycosis has been reported.[6]

In our patient, the clinical course and imaging results of the myocardial infarction are most consistent with coronary plaque rupture possibly provoked by severe vaso-invasive pulmonary infection and multi-organ failure. Microscopy did not show zygomycosis in the coronary arteries.

In a retrospective clinicopathologic study between 1973-2005 among 4396 Japanese patients, Chinen et al. found $50(1.1 \%)$ cases of cardiac fungal infection (CFI)

Exclusive cardiac involvement was observed in only 5 cases (10\%). Kidneys and lungs were the most common co-infected organs. Macroscopic evidence of cardiac involvement consisted of myocardial abscesses $(n=12)$, myocardial hemorrhage $(n=10)$, valvular vegetations $(n=6)$ and mural thrombus $(n=3)$. Epicardial lesions, fibrinous pericarditis and pericardial adhesion were also observed. In 22 cases, the heart was macroscopically grossly unremarkable. Microscopically, the myocardium was the most frequent site of cardiac fungal infection $(n=45)$. Endocardial and epicardial involvement was demonstrated in 10 and 6 cases, respectively. Epicardial involvement directly contiguous with extracardiac infectious lesions were observed in only one case. As in the majority of cases, multiple organs were affected. Major predisposing conditions in this study were malignant neoplasms, hematological disorders and the use of antibiotics and/or corticosteroids. ${ }^{[16]}$

Postmortem diagnosis of cardiac zygomycosis is difficult; however, as in a recent study in India histopathology and cultures were positive in only $73,1 \%$ and $62,4 \%$ of proven 485 cases respectively. ${ }^{[17]}$

Risk factors for zygomycosis 
Risk factors for disseminated zygomycosis include allogenic STC or solid organ transplantation, diabetes mellitus, renal failure, prolonged use of corticosteroids or immunosuppressives, cytotoxic chemotherapy, solid organ transplantation, diabetic ketoacidosis, prolonged neutropenia, infant prematurity, iron overload and use of deferoxamine $\left..^{[1,} \quad 4, \quad 5, \quad 8, \quad 18-21\right] \quad$ Worldwide hematological malignancy tends to be the most common risk factor for zygomycosis. ${ }^{[2]}$ Zygomycosis in immunocompetent individuals is rare but has been described.[20, 22]

Iron overload has been suggested as a risk factor invasive fungal infections including mucormycosis. Hematologic stem cell recipients may be at risk for iron overload if they received multiple erythrocyte transfusions ${ }^{[23,24]}$ Literature shows a tendency for a higher prevalence in males. ${ }^{[25-27]}$

In a recent analysis of ninety-two cases of proven pulmonary mucormycosis (12 from their hospital, rest from literature), hematological disorders $(40.2 \%$, of which $54 \%$ were neutropenic and $29,7 \%$ were on antifungal therapy) and diabetes mellitus (35.9\%) were the most common underlying disease. In $12 \%$ no underlying disease was known. Rhizopus spp was the most common genera, with Mucor second. Fever and cough were the most common clinical symptoms with $69.6 \%$ and 53.3 respectively, hemoptysis was present in $28.3 \%$. Extrapulmonary involvement was found in $16,3 \%$ of patients, with 6 cases of thoracic involvement. Overall survival in this study was $69.6 \% .{ }^{[5]}$

\section{Treatment}

Drug penetration in infected necrotized tissue is poor and mandates surgical debridement as a part of treatment, with surgical treatment independently associated with better outcome than medical treatment alone. ${ }^{[17,28]}$ No specific treatment regime has been reported for cardiac zygomycosis.
Amphotericin B (AmB) formulations are classically the treatment of choice in zygomycosis, however newer triazole agents (posaconazole, isavuconazole) are available for treatment. AmB tends to be the antifungal agent of choice in treatment of zygomycosis, with a preference for liposomal formulations with their better safety profile enabling a longer duration of treatment. Posaconazole and isavuconazole are interesting alternatives due to good in vitro activity against Mucorales and an attractive safety profile. ${ }^{[5,}{ }^{28]}$ However, breakthrough invasive zycomycosis under Posaconazole and itraconazole had been reported. ${ }^{[2]}$

\section{Outcome}

Outcome of zygomycosis is generally poor. Patel et al. reported a 90 -day mortality of $52 \%$ among 465 patients with zygomycosis in India. The median duration of symptoms before admissions was 12 (7-30) days. ${ }^{[17]}$ Mortality rates for disseminated zygomycosis are reported over $90 \% .{ }^{[25,29]}$

In a series of 199 patients with invasive fungal infection after HSCT, total mortality was $46,7 \%$ during a 12-week observation period. Mortality in patients with zygomycosis was $64,3 \%$, for invasive Candidiasis and invasive Aspergillosis $48,9 \%$ and $35,5 \%$ respectively. Nonmyeloablative conditioning and absence of mechanical ventilation and/or hemodialysis were associated with better survival after 6 weeks in invasive fungal infections. ${ }^{[30]}$ In another case series of 41 patients with zygomycosis after HSCT mortality was $48,8 \%$, with $36,6 \%$ directly attributable to invasive zygomycosis. Remarkably one patient in this series died of myocardial infarction. ${ }^{[2]}$

In a recent review of 851 cases reported mortality was $41.0 \%$. Previous hematopoietic stem cell transplantation was the only underlying condition associated with increased 90-day mortality (odds ratio $3.77,95 \% \mathrm{Cl} 1.37-10.37, \mathrm{P}$ $=0.010) \cdot{ }^{[28]}$ Outcome in hematological patients is worse if the start of antifungal therapy is delayed. ${ }^{[31]}$ 


\section{Conclusion}

Zygomycosis is common in immunecompromised patients, especially after HSCT. Mucor species have devastating vaso-invasive properties causing hematogenic dissemination. Antemortem diagnosis may be difficult due to negative cultures. Despite adequate treatment outcome tends to be poor. Cardiac zygomycosis is rare. In our presented patient hematogenic dissemination caused multiple septic embolies resulting in multi organ failure and subsequent myocardial infarction.

Special thanks to Prof. R Nijveldt and D. Bosboom for their contributions and comments on the manuscript.

\section{Conflicts of interest}

None of the authors has any relevant conflicts of interest concerning this case report.

\section{References}

[1]. Freifeld, A. and P. Iwen, Zygomycosis. Semin Respir Crit Care Med, 2004. 25: p. 221-31.

[2]. Ruping, M.J., et al., Forty-one recent cases of invasive zygomycosis from a global clinical registry. J Antimicrob Chemother, 2010. 65(2): p. 296-302.

[3]. Chen, Q., et al., Disseminated mucormycosis with cerebellum involvement due to Rhizomucor pusillus in a patient with multiple myeloma and secondary myelodysplastic syndrome: $A$ case report. Exp Ther Med, 2019. 18(5): p. 4076-4080.

[4]. Rawlinson, N.J., et al., Disseminated Rhizomucor pusillus causing early multiorgan failure during hematopoietic stem cell transplantation for severe aplastic anemia. J Pediatr Hematol Oncol, 2011. 33(3): p. 235-7.

[5]. Feng, J. and X. Sun, Characteristics of pulmonary mucormycosis and predictive risk factors for the outcome. Infection, 2018. 46(4): p. 503-512.

[6]. Mehta, N.N., J. Romanelli, and M.G. Sutton, Native aortic valve vegetative endocarditis with Cunninghamella. Eur J Echocardiogr, 2004. 5(2): p. 156-8.

[7]. Webb, B.J., et al., Concurrent pulmonary Aspergillus fumigatus and mucor infection in a cardiac transplant recipient: a case report. Transplant Proc, 2013. 45(2): p. 792-7.

[8]. Nolan, R.L., et al., Subacute disseminated mucormycosis in a diabetic male. Am J Med Sci, 1989. 298(4): p. 252-5.
[9]. Tansir, G., et al., Disseminated mucormycosis: A sinister cause of neutropenic fever syndrome. Intractable Rare Dis Res, 2017. 6(4): p. 310-313.

[10]. Krishnappa, D., et al., Cardiac mucormycosis: a case report. Eur Heart J Case Rep, 2019. 3(3).

[11]. Jackman, J.D., Jr. and R.L. Simonsen, The clinical manifestations of cardiac mucormycosis. Chest, 1992. 101(6): p. 1733-6.

[12].Nam, Y., et al., Disseminated mucormycosis with myocardial involvement in a renal transplant recipient. Transpl Infect Dis, 2015. 17(6): p. 890-6.

[13]. Ferreira, D., et al., Acute myocardial infarction in disseminated mucormycosis infection. Eur Heart J, 2017.38(11): p. 838.

[14]. Virmani, R., D.H. Connor, and H.A. McAllister, Cardiac mucormycosis. A report of five patients and review of 14 previously reported cases. Am J Clin Pathol, 1982. 78(1): p. 42-7.

[15]. Sanchez-Recalde, A., et al., Successful treatment of prosthetic aortic valve mucormycosis. Chest, 1999. 116(6): p. 1818-20.

[16]. Chinen, K., et al., Fungal infections of the heart: a clinicopathologic study of 50 autopsy cases. Pathol Res Pract, 2007. 203(10): p. 705-15.

[17]. Patel, A., et al., A multicentre observational study on the epidemiology, risk factors, management and outcomes of mucormycosis in India. Clin Microbiol Infect, 2020. 26(7): p. 944 e9-944 e15.

[18]. Ibrahim, A.S., B. Spellberg, and J. Edwards, Jr., Iron acquisition: a novel perspective on mucormycosis pathogenesis and treatment. Curr Opin Infect Dis, 2008. 21(6): p. 620-5.

[19]. St-Germain, G., et al., Infection due to Rhizomucor pusillus: report of four cases in patients with leukemia and review. Clin Infect Dis, 1993. 16(5): p. 640-5.

[20]. Kimura, M., et al., Isolation and identification of Rhizomucor pusillus from pleural zygomycosis in an immunocompetent patient. Med Mycol, 2009. 47(8): p. 869-73.

[21]. Hosseini, S.M. and P. Borghei, Rhinocerebral mucormycosis: pathways of spread. Eur Arch Otorhinolaryngol, 2005. 262(11): p. 932-8.

[22]. Hussain, S., et al., Rhinocerebral invasive mycosis: occurrence in 
immunocompetent individuals. Eur J Radiol, 1995. 20(2): p. 151-5.

[23].Hassan, W., et al., Enhancement of ironcatalyzed lipid peroxidation by acidosis in brain homogenate: comparative effect of diphenyl diselenide and ebselen. Brain Res, 2009. 1258: $\mathrm{p}$. 71-7.

[24].Kontoyiannis, D.P., et al., Increased bone marrow iron stores is an independent risk factor for invasive aspergillosis in patients with high-risk hematologic malignancies and recipients of allogeneic hematopoietic stem cell transplantation. Cancer, 2007. 110(6): p. 1303-6.

[25].Roden, M.M., et al., Epidemiology and outcome of zygomycosis: a review of 929 reported cases. Clin Infect Dis, 2005. 41(5): p. 634-53.

[26].Lee, F.Y., S.B. Mossad, and K.A. Adal, Pulmonary mucormycosis: the last 30 years. Arch Intern Med, 1999. 159(12): p. 1301-9.

[27].Tedder, M., et al., Pulmonary mucormycosis: results of medical and surgical therapy. Ann Thorac Surg, 1994. 57(4): p. 104450.

[28].Jeong, W., et al., The epidemiology and clinical manifestations of mucormycosis: a systematic review and metaanalysis of case reports. Clin Microbiol Infect, 2019. 25(1): p. 26-34.

[29].Spellberg, B., J. Edwards, Jr., and A. Ibrahim, Novel perspectives on mucormycosis: pathophysiology, presentation, and management. Clin Microbiol Rev, 2005. 18(3): p. 556-69.

[30].Neofytos, D., et al., Epidemiology and outcome of invasive fungal infection in adult hematopoietic stem cell transplant recipients: analysis of Multicenter Prospective Antifungal Therapy (PATH) Alliance registry. Clin Infect Dis, 2009. 48(3): p. 265-73.

[31].Chamilos, G., R.E. Lewis, and D.P. Kontoyiannis, Delaying amphotericin B-based frontline therapy significantly increases mortality among patients with hematologic malignancy who have zygomycosis. Clin Infect Dis, 2008. 47(4): p. 503-9. 